\title{
Neuropeptide Y-based recombinant peptides ameliorate bone loss in mice by regulating hematopoietic stem/progenitor cell mobilization
}

\author{
Min Hee Park ${ }^{1,2,3}$, Namoh Kim ${ }^{1,2,3}$, Hee Kyung Jin ${ }^{1,4, *}$ E Jae-sung Bae $e^{1,2,3, *}$ \\ ${ }^{1}$ Stem Cell Neuroplasticity Research Group, Kyungpook National University, Daegu 41566, ${ }^{2}$ Department of Physiology, Cell and Matrix \\ Research Institute, School of Medicine, Kyungpook National University, Daegu 41944, ${ }^{3}$ Department of Biomedical Science, BK21 Plus \\ KNU Biomedical Convergence Program, Kyungpook National University, Daegu 41944, ${ }^{4}$ Department of Laboratory Animal Medicine, \\ College of Veterinary Medicine, Kyungpook National University, Daegu 41566, Korea
}

\begin{abstract}
Ovariectomy-induced bone loss is related to an increased deposition of osteoclasts on bone surfaces. We reported that the 36-amino-acid-long neuropeptide Y (NPY) could mobilize hematopoietic stem/progenitor cells (HSPCs) from the bone marrow to the peripheral blood by regulating HSPC maintenance factors and that mobilization of HSPCs ameliorated low bone density in an ovariectomy-induced osteoporosis mouse model by reducing the number of osteoclasts. Here, we demonstrated that new NPY peptides, recombined from the cleavage of the full-length NPY, showed better functionality for HSPC mobilization than the full-length peptide. These recombinant peptides mediated HSPC mobilization with greater efficiency by decreasing HSPC maintenance factors. Furthermore, treatment with these peptides reduced the number of osteoclasts and relieved ovariectomy-induced bone loss in mice more effectively than treatment with full-length NPY. Therefore, these results suggest that peptides recombined from full-length NPY can be used to treat osteoporosis. [BMB Reports 2017; 50(3): 138-143]
\end{abstract}

\section{INTRODUCTION}

Osteoporosis is a systemic skeletal disease characterized by low bone density and degeneration of the microarchitecture of bone tissue (1-3). The pathogenesis of osteoporosis stems from an imbalance between bone formation by osteoblasts and

*Corresponding authors. Jae-sung Bae, Tel: +82-53-420-4815; Fax: +82-53-424-3349; E-mail: jsbae@knu.ac.kr, Hee Kyung Jin, Tel: +82-53-950-5966; Fax: +82-53-950-5955; E-mail: hkjin@knu.ac.kr

https://doi.org/10.5483/BMBRep.2017.50.3.191

Received 15 November 2016, Revised 9 December 2016, Accepted 19 December 2016

Keywords: Bone loss, Hematopoietic stem/progenitor cell, Mobilization, Neuropeptide Y-based recombinant peptides, Osteoclasts bone resorption by osteoclasts. Specifically, excessive deposition of osteoclasts on bone surfaces is one of the major causes of osteoporosis $(4,5)$. Osteoclasts are specialized cells that degrade bone in order to initiate normal bone remodeling and mediate bone loss in pathological conditions. Osteoclasts differentiate from hematopoietic stem/progenitor cells (HSPCs) in the bone marrow (BM) (6). HSPCs reside in specialized environments known as stem cell niches in the BM, which consist of supporting cells that regulate HSC survival, proliferation, and mobilization (7-9). The niche cells that maintain HSPCs in the BM express various hematopoietic cytokines and adhesion molecules, such as stromal cellderived factor 1 alpha (SDF-1 $\alpha$ ), stem cell factor Kit-ligand (Kitl), angiopoietin-1 (Angtp1), vascular cell adhesion molecule 1 (Vcam1), interleukin-7 (IL7), and secreted phosphoprotein 1 (Spp1) (10-14). The reduction in expression of these factors results in induction of HSPC mobilization $(9,15)$.

Previous studies have demonstrated that AMD3100-induced SDF-1 $\alpha$ can induce HSPC mobilization and ameliorate bone loss in an ovariectomy-induced osteoporosis mouse model by decreasing the number of osteoclasts differentiated from BM-derived HSPCs (16). Moreover, recruitment of osteoclast precursors from the $\mathrm{BM}$ to the blood relieves bone loss in ovariectomized mice by reducing osteoclast accumulation on bone surfaces (17). Neuropeptide Y (NPY) is known to regulate a variety of physiological processes such as appetite, energy storage, and pain (18-20). Our recent study demonstrated a new role for NPY in the BM $(21,22)$. Notably, NPY induced HSPC mobilization through the regulation of HSPC maintenance factors, leading to amelioration of bone loss in ovariectomized mice by reducing osteoclast deposition (22). Moreover, a previous study reported that neprilysin-cleaved NPY fragments had a greater neuroprotective effect in neurodegenerative disease than did the full-length 36-amino-acid-long NPY peptide (23).

This study was designed to identify functional recombinant peptides from full-length NPY with the capability to efficiently mobilize HSPCs and prevent bone loss in ovariectomized mice. 
We found two NPY-based recombinant peptides with greater beneficial effects against ovariectomy-induced osteoporosis in mice than that of the full-length NPY, suggesting their potential therapeutic application in the treatment of osteoporosis.

\section{RESULTS}

\section{NPY (6-20) and NPY (21-36) recombined from full-length NPY induce HSPC mobilization}

To search for more effective sequences from the full-length NPY for HSPC mobilization, we recombined full length NPY (1-36) into four different peptides: NPY (1-15), NPY (6-20), NPY (11-25), and NPY (21-36). Recent studies demonstrated that HSPC maintenance factors such as SDF-1 $\alpha$, Kitl, Angpt1, Vcam1, IL7, and Spp1 (10-14) are important for maintaining HSPCs with niche cells in the BM, and a reduction of these factors induces HSPC mobilization $(15,24)$. Moreover, NPY (1-36) induces HSPC mobilization by reducing these factors in the BM (22). Therefore, we confirmed the expression of these factors in the BM of mice treated with NPY (1-15), NPY (6-20), NPY (11-25), or NPY (21-36). The four different peptides decreased the expression of SDF-1 $\alpha$ in the BM compared to in PBS-treated mice. Particularly, the mice treated with NPY (6-20) or NPY (21-36) showed greater reductions of Kitl, Angpt1, IL7, and Spp1 than did mice treated with NPY (1-36)

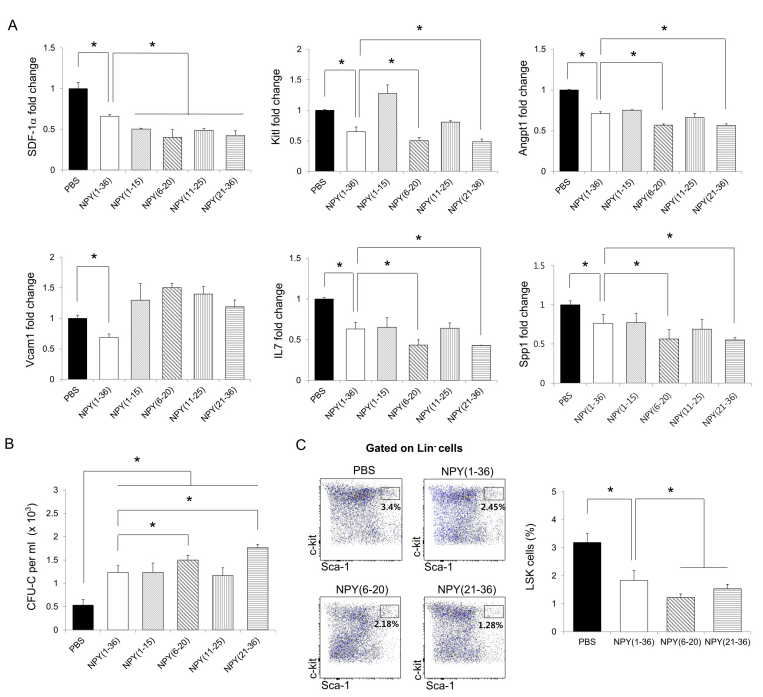

Fig. 1. Neuropeptide Y-based recombinant peptides induces HSPC mobilization in vivo. (A and B) Expression of (A) SDF-1 $\alpha$, Angpt1, IL7, Kitl, Vcam1, and Spp1 in BM and (B) the number of hematopoietic CFÚ cells in the PB of WT mice treated with PBS, full-length NPY (1-36), NPY (1-15), NPY (6-21), NPY (11-25), or NPY (21-36) ( $\mathrm{n}=3$ per group). (C) Flow cytometry analysis of LSK cells in the BM of WT mice treated with PBS, NPY (1-36), NPY (6-21), or NPY (21-36) ( $n=3-4$ per group). ${ }^{*} \mathrm{P}<0.05$. All error bars indicate S.E.M. All expression levels are relative to that of GAPDH mRNA.
(Fig. 1A).

Next, we performed a colony-forming unit assay (CFU-C) to determine whether these peptides could mobilize HSPCs from the $\mathrm{BM}$ into the peripheral blood (PB). As shown in Fig. 1B, all peptides induced HSPC mobilization. Strikingly, NPY (21-36) resulted in the greatest increase in hematopoietic CFU cells in the PB compared with the other three peptides. Based on the results shown in Figs. $1 A$ and $B$, we used flow cytometry to reconfirm the ability of NPY (6-20) and NPY (21-36) to mobilize HSPCs in the BM. As expected, the percentage of $\mathrm{Lin}^{-} \mathrm{Sca}-1^{+} \mathrm{C}-\mathrm{Kit}^{+}$(LSK) cells was considerably reduced in the BM of NPY (6-20) or NPY (21-36)-treated mice compared to that in NPY (1-36)-treated mice (Fig. 1C), indicating that NPY (6-20) and NPY (21-36) could effectively egress HSPCs from the BM into the PB by regulating HSPC maintenance factors.

Osteoblasts, which are important cells that maintain HSPCs in the BM, express HSPC maintenance factors $(9,15)$. NPY (1-36)-induced HSPC mobilization occurs by the regulation of these factors in osteoblasts (22). To investigate whether NPY (6-20) and NPY (21-36) could decrease these factors in osteoblasts, we first cultured osteoblasts differentiated from BM-derived mesenchymal stromal cells (MSCs) and then treated the cells with NPY (6-20) or NPY (21-36) for 3 days (Fig. 2A). These two peptides also caused a greater reduction

A

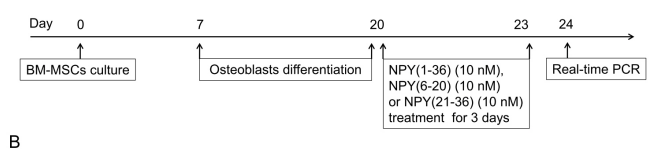

B
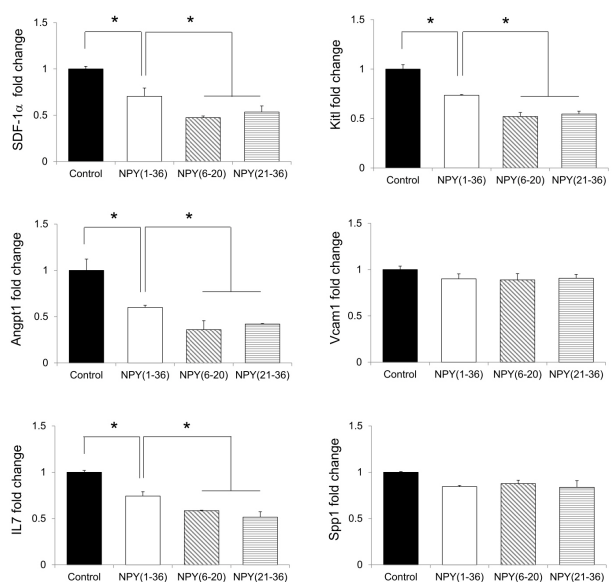

Fig. 2. Neuropeptide Y-based NPY (6-20) and NPY (21-36) reduces HSPC maintenance factors in osteoblasts in vitro. (A) Experimental design used to investigate the effect of NPY (6-20) or NPY (21-36) on HSPC maintenance factors in osteoblasts. (B) Expression levels of SDF-1 $\alpha$, Kitl, Angpt1, Vcam1, Spp1, and IL7 in osteoblasts treated with NPY (1-36), NPY (6-20), or NPY (21-36) ( $\mathrm{n}=3$ per group). ${ }^{*} \mathrm{P}<0.05$. All error bars indicate S.E.M. All expression levels are relative to that of GAPDH mRNA. 
in HSPC maintenance factors in osteoblasts than did NPY (1-36) (Fig. 2B). Taken together, these results suggest that NPY (6-20) and NPY (21-36) induce HSPC mobilization more effectively.

\section{NPY (6-20) and NPY (21-36) relieves ovariectomy-induced bone loss by inducing HSPC mobilization}

A previous study reported that sphingosine-1-phosphate, which egresses osteoclast precursors from the BM into the PB, prevents bone loss in ovariectomized mice by reducing osteoclast deposition on bone surfaces (17). NPY (1-36)-induced HSPC mobilization also ameliorates bone loss in ovariectomized mice by reducing the number of osteoclasts in the BM (22). Based on our results, we predicted that NPY (6-20) and NPY (21-36) could more effectively protect against bone loss in the ovariectomy-induced osteoporosis mouse model than NPY (1-36) treatment. To investigate this hypothesis, we first examined the effects of NPY (6-20) and NPY (21-36) on HSPC mobilization in ovariectomized mice. Twelve-week-old female mice were first subjected to either sham surgery or

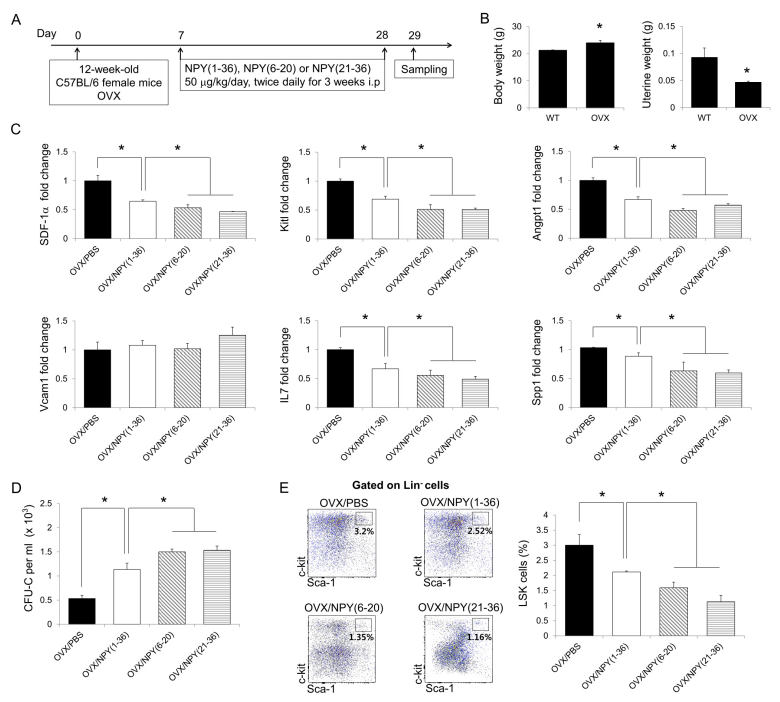

Fig. 3. Neuropeptide Y-based NPY (6-20) and NPY (21-36) egress HSPCs from the $\mathrm{BM}$ into the $\mathrm{PB}$ in ovariectomized mice. (A) Experimental design to determine the effects of NPY (6-20) and NPY (21-36) in ovariectomized mice. Twelve-week-old female WT mice were subjected to ovariectomy (OVX). One week after surgery, ovariectomized mice received an intraperitoneal injection with PBS, NPY (1-36), NPY (6-20), or NPY (21-36) for 21 days. (B) Body (left) and uterine (right) weights were measured to confirm the success of ovariectomy ( $\mathrm{n}=3-5$ mice per group). (C and D) Expression levels of (C) HSPC maintenance factors in the $\mathrm{BM}$ and (D) the number of CFU cells in the PB of ovariectomized mice treated with PBS, NPY (1-36), NPY (6-20), or NPY (21-36) ( $n=3$ mice per group). (E) Flow cytometry analysis of LSK cells in the BM of each group ( $n=5-7$ mice per group). $* P<0.05$. All error bars indicate S.E.M. All expression levels are relative to that of GAPDH mRNA. ovariectomy. At one week after surgery, these mice received an i.p. injection with PBS, NPY (1-36), NPY (6-20), or NPY (21-36) for 21 days (Fig. 3A). To ensure the success of the ovariectomy procedure, we checked the body and uterine weights of the ovariectomized mice. The results revealed elevated body weights and decreased uterine weights in the ovariectomized mice compared to in sham-operated mice (Fig. 3B). HSPC maintenance factors were markedly decreased in the BM of NPY (6-20) and NPY (21-36)-treated ovariectomized mice, and hematopoietic progenitors and LSK cells were mobilized (Fig. 3C-E).

Next, the femurs of ovariectomized mice were analyzed by $\mu C T$ after NPY (6-20) or NPY (21-36) treatment. Bone density showed a greater increase in mice treated with either NPY (6-20) or NPY (21-36) than in those treated with NPY (1-36) (Fig. 4A). Compared to NPY (1-36) treatment, NPY (6-20) or NPY (21-36) treatment also resulted in higher increases in the bone volume/total volume (BV/TV) and trabecular thickness (Tb. Th), and higher decreases in trabecular spacing (Tb. S) (Fig. 4B). To determine whether HSPC mobilization by NPY (6-20) and NPY (21-36) affected osteoclast reduction, we

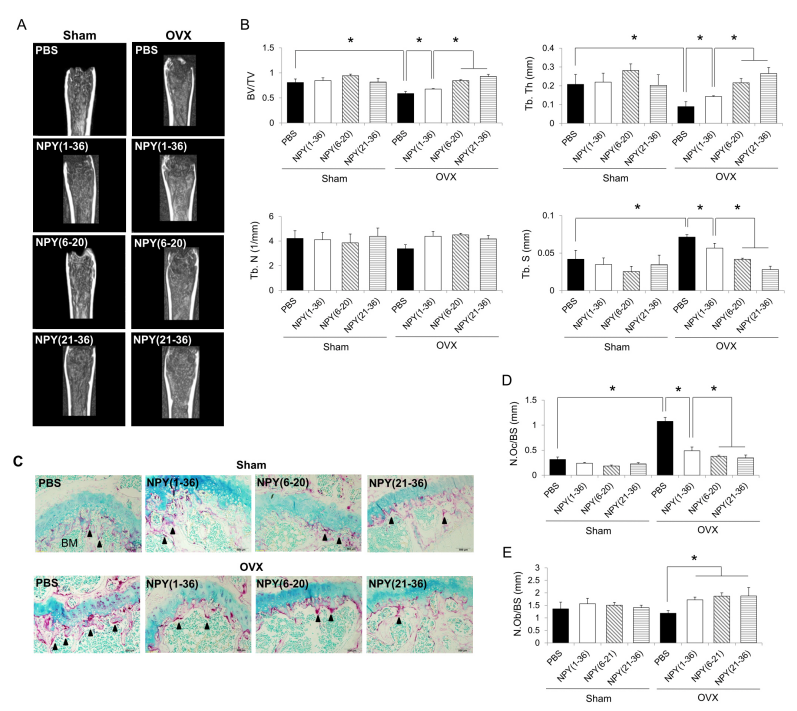

Fig. 4. Neuropeptide Y-based NPY (6-20) or NPY (21-36) prevents bone loss in ovariectomized mice. (A) Representative $\mu \mathrm{CT}$ images of the distal femurs from sham or ovariectomized mice treated with PBS, NPY (1-36), NPY (6-20), or NPY (21-36). (B) Bone volume/total volume (BV/TV), trabecular thickness (Tb. Th), trabecular number (Tb. N), and trabecular spacing (Tb. S) calculated from $2.5 \times 0.5 \times 0.5 \mathrm{~mm}^{3}$ of the distal femoral bone ( $\mathrm{n}=3-5$ mice per group). (C) Representative pictures showing osteoclasts in the trabecular region. Arrowheads indicate active tartrate-resistant acid phosphatase $\left(\mathrm{TRAP}^{+}\right)$-positive osteoclasts stained in red (original magnification, 200×). (D and E) Histogram representing the (D) osteoclast number/bone surface (N. Oc/BS) and $(\mathrm{E})$ osteoblast number/bone surface $(\mathrm{N}$. Ob/BS) $(\mathrm{n}=3-5$ mice per group). ${ }^{*} \mathrm{P}<0.05$. All error bars indicate S.E.M. All expression levels are relative to that of GAPDH mRNA. 
performed tartrate-resistant acid phosphatase (TRAP) staining in the trabecular region of each group. The number and size of TRAP + active osteoclasts (black arrowhead) were decreased in the trabecular region after NPY (6-20) or NPY (21-36) treatment (Fig. 4C and D). Consistent with our findings, treatment with either of these two peptides caused a greater reduction in osteoclast deposition than did NPY (1-36) treatment. These results indicate that NPY (6-20) or NPY (21-36) treatment shows better protection against bone loss in ovariectomized mice than does NPY (1-36) treatment.

We also performed hematoxylin and eosin staining to determine the effects of these peptides on the number of osteoblasts. The results showed significantly increased numbers of osteoblasts in the BM of NPY (6-20) or NPY (21-36)-treated ovariectomized mice, which was similar to results of NPY (1-36) treatment (Fig. 4E). Therefore, our findings suggest that either NPY (6-20) or NPY (21-36) treatment egresses HSPCs from the BM into the PB, leading to an amelioration of bone loss in ovariectomized mice by decreasing the number of osteoclasts attached to the bone surface, as well as by increasing the number of osteoblasts. Furthermore, NPY (6-20) and NPY (21-36) are more effective than full-length NPY peptide in protecting against ovariectomyinduced osteoporosis through HSPC mobilization.

\section{DISCUSSION}

Previous studies have suggested the mobilization of osteoclast precursors or HSPCs that differentiate into osteoclasts can be used to treat osteoporosis $(16,17)$. Recently, NPY was shown to mobilize HSPCs by reducing the expression of HSPC maintenance factors in osteoblasts, resulting in an amelioration of ovariectomy-induced bone loss by reducing osteoclast deposition on bone surfaces (22). However, the specific sequences of NPY that induce HSPC mobilization have not been characterized. To identify the active sequences of NPY, we produced NPY-based recombinant peptides from the full-length NPY peptide.

We found that both NPY (6-20) and NPY (21-36) decreased the expression of HSPC maintenance factors (such as SDF-1 $\alpha$, Kitl, Angpt1, IL7, and Spp1) in osteoblasts better than that by full-length NPY. Particularly, using the CFU-C assay (which estimates HSPC egress from the BM into the PB), NPY (21-36) was shown to elicit the highest increase in the number of hematopoietic CFU cells in the PB. We also confirmed that the LSK compartment was decreased in the BM by administration of either NPY (6-20) or NPY (21-36). These results suggest that NPY (6-20) and NPY (21-36) can induce HSPC mobilization more effectively than full-length NPY and that these peptides contain the active sequences for inducing HSPC mobilization.

Treatment with either NPY (6-20) or NPY (21-36) in the ovariectomy-induced osteoporosis mouse model also resulted in more efficient HSPC mobilization effect than did full-length NPY treatment. Consistent with these results, $\mu \mathrm{CT}$ analyses revealed that bone thickness increased because of the inhibition of osteoclastic bone resorption. These results indicate that NPY (6-20) and NPY (21-36) prevent bone loss in ovariectomized mice by reducing the number of osteoclasts deposited on bone surfaces, and that their ability to treat osteoporosis is more effective than that of full-length NPY.

In summary, we demonstrated the characteristic role of NPY-based recombinant peptides on HSPC mobilization and their improved amelioration of bone loss in ovariectomized mice over the original effect of full-length NPY. NPY has the therapeutic benefit of being a stable peptide that is synthesized in the human body. Therefore, these peptides recombined from full-length NPY are safe. Moreover, these peptides contain fewer amino acids (15-16 amino acids on average) than full-length NPY, suggesting that they can be inexpensively produced using recombinant technology. In conclusion, our data suggest the efficient use of new peptides recombined from full-length NPY for therapeutic intervention for osteoporosis.

\section{MATERIAIS AND METHODS}

\section{Mice}

Six- to eight-week-old male or female C57BL/6 mice were purchased from the Jackson Laboratory (Bar Harbor, ME, USA). The block randomization method was used to allocate the animals to experimental groups. To eliminate bias, investigators were blinded during data collection and data analysis. Mice were housed with a 12-h day-night cycle and free access to tap water and food pellets. All mouse studies were approved by the Kyungpook National University Institutional Animal Care and Use Committee.

\section{Reagents treatment}

To examine the effects of NPY-based recombinant peptides on HSPC mobilization, the full-length NPY (NPY1-36, H-6375; Bachem, Bubendorf, Switzerland) was cleaved and recombined to NPY (1-15), NPY (6-20), NPY (11-25), and NPY (21-36), which were all from Peptron, Inc. (Daejeon, Korea). Under isoflurane anesthesia, wild-type (WT) mice were injected intravenously with $50 \mu \mathrm{g} / \mathrm{kg}$ body weight of NPY (1-36), NPY (1-15), NPY (6-20), NPY (11-25), or NPY (21-36). After $1 \mathrm{~h}$, the blood and BM were harvested and analyzed. For in vitro experiments, $10 \mathrm{nM}$ each of full-length NPY, NPY (1-15), NPY (6-20), NPY (11-25), or NPY (21-36) was applied to osteoblasts differentiated from BM-MSCs. Three days later, the cells were collected for RNA extraction.

\section{Hematopoietic colony-forming cell unit assay}

Single-cell suspensions of peripheral blood lysed with $0.15 \mathrm{M}$ $\mathrm{NH}_{4} \mathrm{Cl}$ (StemCell Technologies, Vancouver, BC, Canada) were plated into $35-\mathrm{mm}$ dishes $\left(3 \times 10^{5}\right.$ cells/plate) with MethoCult GF M3434 (StemCell Technologies). Hematopoietic colonies were counted and scored after incubating the plates for 12-14 
days at $37^{\circ} \mathrm{C}$ and $5 \% \mathrm{CO}_{2}$ according to the manufacturer's instructions.

\section{Flow cytometry}

The BM was flushed from the tibia and femur of each mouse. Red blood cells were lysed for 5 min at $4^{\circ} \mathrm{C}$ in $0.15 \mathrm{M} \mathrm{NH}_{4} \mathrm{Cl}$, washed once with phosphate-buffered saline (PBS; Gibco, Grand Island, NY, USA), and then counted using a hemocytometer. For HSPCs, $\mathrm{Lin}^{+}$cells were removed by magnetic depletion using biotinylated lineage-specific antibodies (CD5, CD45R, CD11b, Gr-1, and Ter-119), followed by depletion with MACS beads conjugated to a monoclonal anti-biotin antibody (Miltenyi Biotec, Bergisch Gladbach, Germany). For staining of the HSPCs, Lin $^{-}$cells were stained with phycoerythrin-Cy7-conjugated antibodies to Sca1 (558162) and allophycocyanin-conjugated antibodies to c-Kit (553356), all from BD Biosciences (Franklin Lakes, NJ, USA). Data were collected using BD LSRII and Arialll systems (BD Biosciences) and analyzed using Flowjo software (Tress Star, Ashland, OR, USA).

\section{Osteogenic differentiation of BM-MSC}

Tibias and femurs were dissected from 4- to 6-week-old C57BL6 mice. The BM was harvested, and single-cell suspensions were obtained using a $40-\mu \mathrm{m}$ cell strainer (BD Biosciences). To culture MSCs, approximately $10^{7}$ cells were plated in $75-\mathrm{cm}^{2}$ flasks containing MyeloCult M5300 culture medium (StemCell Technologies) with antibiotics according to our previous reports (25). After 1 week, the medium was replaced with fresh Osteogenic Differentiation Medium (R\&D Systems, Minneapolis, MN, USA), containing mouse Osteogenic Supplement (R\&D Systems) and penicillin-streptomycin to induce osteogenesis, and the media were subsequently changed every 3 days for 2 weeks.

\section{Ovariectomy}

Twelve-week-old female mice were subjected to either sham surgery or ovariectomy. One week after surgery, shamoperated and ovariectomized mice were injected intraperitoneally with $50 \mu \mathrm{g} / \mathrm{kg}$ body weight of NPY (1-36), NPY (1-15), NPY (6-20), NPY (11-25), or NPY (21-36) every $12 \mathrm{~h}$ for 21 days. At $1 \mathrm{~h}$ after the last injection, the mice were sacrificed for analysis.

\section{Bone histomorphometry}

To identify osteoclasts and osteoblasts, bones were fixed overnight in $4 \%$ paraformaldehyde (Sigma, St. Louis, MO, USA) in PBS at $4^{\circ} \mathrm{C}$. The bones were decalcified in $10 \%$ EDTA for up to 5 weeks and embedded in paraffin, and then cut into $5-\mu \mathrm{m}$ sections. Sections were stained with hematoxylin and eosin to visualize the osteoblasts. For osteoclast visualization, sections were stained with $225 \mu \mathrm{M}$ naphthol AS-MX phosphate (Sigma), 0.84\% N,N-dimethylformamide (Sigma), and $1.33 \mathrm{mM}$ Fast Red Violet LB Salt (Sigma) in $50 \mathrm{mM}$ sodium acetate $(\mathrm{pH} 5.0)$ containing $50 \mathrm{mM}$ sodium tartrate and incubated for $30 \mathrm{~min}$. After incubation, the sections were washed in distilled water and counterstained with $1 \%$ methyl green. We performed histomorphometric analysis using the Bioquant OSTEOII Program (Bioquant Image Analysis Corporation, Nashville, TN, USA). For $\mu \mathrm{CT}$ imaging, the femurs of mice were fixed and prepared for analysis. $\mu \mathrm{CT}$ imaging was performed on a $\mu \mathrm{CT}$ scanner (Inveon Preclinical CT, Siemens Healthcare, Hoffman Estates, IL, USA) at 40- $\mu \mathrm{m}$ slice thickness with an exposure time of $600 \mathrm{~ms}$, photon energy of $70 \mathrm{keV}$, and current of $400 \mu \mathrm{A}$. The bone parameters (bone volume/total volume, bone surface area/bone volume, trabecular thickness, trabecular number, and trabecular spacing) were calculated from a $2.5 \times 0.5 \times 0.5 \mathrm{~mm}^{3}$ area of the distal femoral bone using the Siemens Inveon Software.

\section{Quantitative real-time PCR}

RNA was extracted from BM using the RNeasy Lipid Tissue Mini kit (Qiagen) according to the manufacturer's instructions. cDNA was synthesized from $5 \mu \mathrm{g}$ of total RNA using a commercially available kit from Clontech (Mountain View, CA, USA). Quantitative real-time PCR was performed using a Corbett Research RG-6000 real-time PCR instrument. The following primers were used; SDF- $1 \alpha$ (forward, 5'-TTCGAT CAGAGCCCATAGAG-3'; reverse, 5'-CCAGACCATCCTGGAT AATG-3'), Kitl (forward, 5'-CCAAAAGCAAAGCCAATTACA AG-3'; reverse, 5'-AGACTCGGGCCTACAATGGA-3'), Angpt1 (forward, 5'-TATTTTGTGATTCTGGTGATT-3'; reverse, 5'-GT TTCGCTTTATTTTTGTAATG-3'), Vcam1 (forward, 5'-AAAAGC GGAGACAGGAGACA-3'; reverse, 5'-AGCACGAGAAGCTCA GGAGA-3'), IL7 (forward, 5'-TCTGGGCAAATGGTGATTAT-3'; reverse, 5'-CCCAACCCCTATAATGCTCT-3'), Spp1 (forward: 5'-GTTGGGGACATCGACTGTAG-3'; reverse, 5'-ATTCTGTG GACTCGGATGAA-3'), and GAPDH (forward, 5'-TGGCAAA GTGGAGATTGTTGCC-3'; reverse, 5'-AAGATGGTGATGGGC TTCCCG-3').

\section{Statistical analysis}

Comparisons between two groups were performed with Student's $t$-test. In cases in which more than two groups were compared, one-way analysis of variance (ANOVA) was used, followed by Tukey's HSD test. All statistical analyses were performed using SPSS statistical software. $\mathrm{P}<0.05$ was considered to indicate statistical significance.

\section{ACKNOWLEDGEMENTS}

This research was supported by Kyungpook National University Bokhyeon Research Fund, 2015.

\section{CONFLICTS OF INTEREST}

The authors have no conflicting financial interests. 


\section{REFERENCES}

1. van den Bergh JP, van Geel TA and Geusens PP (2012) Osteoporosis, frailty and fracture: implications for case finding and therapy. Nat Rev Rheumatol 8, 163-172

2. Manolagas SC (2000) Sex Steroids and Bone. Endocr Rev 21, 115-137

3. NIH Consensus Development Panel on Osteoporosis Prevention, Diagnosis, and therapy (2001) Osteoporosis prevention, diagnosis, and therapy. JAMA 285, 785-795

4. Kim TH, Jung JW, Ha BG et al (2011) The effects of luteolin on osteoclast differentiation, functionin vitro and ovariectomy-induced bone loss. J Nutr Biochem 22, 8-15

5. Kim T, Ha H, Shim KS, Cho WK and Ma JY (2013) The anti-osteoporotic effect of Yijung-tang in an ovariectomized rat model mediated by inhibition of osteoclast differentiation. J Ethnopharmacol 146, 83-89

6. Takayanagi H (2007) Osteoimmunology: shared mechanisms and crosstalk between the immune and bone systems. Nat Rev Immunol 7, 292-304

7. Ding L, Saunders TL, Enikolopov G and Morrison SJ (2012) Endothelial and perivascular cells maintain haematopoietic stem cells. Nature 481, 457-462

8. Butler JM, Nolan DJ, Vertes EL et al (2010) Endothelial cells are essential for the self-renewal and repopulation of Notch-dependent hematopoietic stem cells. Cell Stem Cell 6, 251-264

9. Lévesque JP, Helwani FM and Winkler IG (2010) The endosteal 'osteoblastic' niche and its role in hematopoietic stem cell homing and mobilization. Leukemia 24, 19791992

10. Arai F, Hirao A, Ohmura M et al (2004) Tie2/angiopoietin-1 signaling regulates hematopoietic stem cells quiescence in the bone marrow niche. Cell 118, 149-161

11. Sugiyama $T$, Kohara $H$, Noda $M$ and Nagasawa $T$ (2006) Maintenance of the hematopoietic stem cell pool by CXCL12-CXCR4 chemokine signaling in bone marrow stromal cell niches. Immunity 25, 977-988

12. Tanaka Y, Morimoto I, Nakano Y et al (1995) Osteoblasts are regulated by the cellular adhesion through ICAM-1 and VCAM-1. J Bone Miner Res 10, 1462-1469

13. Jung $Y$, Wang J, Schneider A et al (2006) Regulation of SDF-1 (CXCL12) production by osteoblasts; a possible mechanism for stem cell homing. Bone 38, 497-508
14. Méndez-Ferrer S, Michurina TV, Ferraro F et al (2010) Mesenchymal and haematopoietic stem cells form a unique bone marrow niche. Nature 466, 829-834

15. Katayama Y, Battista M, Kao WM et al (2006) Signals from the sympathetic nervous system regulate hematopoietic stem cell egress from bone marrow. Cell 124, 407-421

16. Im JY, Min WK, Park MH et al (2014) AMD3100 improves ovariectomy-induced osteoporosis in mice by facilitating mobilization of hematopoietic stem/progenitor cells. BMB Rep 47, 439-444

17. Ishii M, Egen JG, Klauschen F et al (2009) Sphingosine-1phosphate mobilizes osteoclast precursors and regulates bone homeostasis. Nature 458, 524-528

18. Zukowska-Grojec Z (1995) Neuropeptide Y. A novel sympathetic stress hormone and more. Ann NY Acad Sci $771,219-233$

19. Kalra SP and Kalra PS (2004) NPY and cohorts in regulation appetite, obesity and metabolic syndrome: beneficial effects of gene therapy. Neuropeptides 38, 201-211

20. Kuo LE, Kitlinska JB, Tilan JU et al (2007) Neuropeptide $Y$ acts directly in the periphery on fat tissue and mediates stress-induced obesity and metabolic syndrome. Nat Med 13, 803-811

21. Park MH, Jin HK, Min WK et al (2015) Neuropeptide $Y$ regulates the hematopoietic stem cell microenvironment and prevents nerve injury in the bone marrow. EMBO J 34, 1648-1660

22. Park MH, Lee JK, Kim N et al (2016) Neuropeptide $Y$ Induces Hematopoietic Stem/Progenitor Cell Mobilization by Regulating Matrix Metalloproteinase-9 Activity Through Y1 Receptor in Osteoblasts. Stem Cells 34, 2145-2156

23. Rose JB, Crews L, Rockenstein E et al (2009) Neuropeptide $Y$ fragments derived from neprilysin processing are neuroprotective in a transgenic model of Alzheimer's disease. J Neurosci 29, 1115-1125

24. Ehninger A and Trumpp A (2011) The bone marrow stem cell niche grows up: mesenchymal stem cells and macrophages move in. J Exp Med 208, 421-428

25. Bae JS, Furuya S, Shinoda Y et al (2005) Neurodegeneration augments the ability of bone marrow-derived mesenchymal stem cells to fuse with Purkinje neurons in Niemann-Pick type C mice. Hum Gene Ther 16, 10061011 